\title{
Some Spectral Properties of Multiplicative Hermite Equation
}

\author{
Sertaç Göktaş ${ }^{1}$, Emrah Yılmaz ${ }^{2 *}$ and Ayşe Çiğdem Yar $^{2}$ \\ ${ }^{1}$ Department of Mathematics, Faculty of Science and Letters, Mersin University, Mersin,Turkey \\ ${ }^{2}$ Department of Mathematics, Faculty of Science, Firat University, Elazı̆̆, Turkey \\ *Corresponding author
}

\author{
Article Info \\ Keywords: Hermite equation, Multi- \\ plicative calculus, Multiplicative Sturm- \\ Liouville equation \\ 2010 AMS: 11N05, 35Q41, 39A20 \\ Received: 19 July 2021 \\ Accepted: 30 January 2022 \\ Available online: 11 February 2022
}

\begin{abstract}
We reconstruct the Multiplicative Hermite Equation from multiplicative Sturm-Liouville equation. A new representation of eigenfunctions for the constructed problem are obtained by the power series solution technique. While making these solutions, multiplicative Hermite polynomials were used strongly. We get a generator for multiplicative Hermite polynomials and construct integration representations for these polynomials. Finally, some spectral properties of the multiplicative Hermite problem are examined in detail.
\end{abstract}

\section{Introduction}

Multiplicative calculus was introduced by Grossman and Katz [1,2] in 1967 as an alternative to usual calculus. This type of calculus is also known as non-Newtonian because of its difference from classical calculus of Newton and Leibniz. Multiplicative calculus is a useful supplement to usual calculus in that it is tailored to situations involving exponential functions in the same sense that the usual calculus is tailored to situations involving linear functions. Multiplicative calculus moves the roles of subtraction and addition to division and multiplication. There are actually many reasons to study multiplicative analysis. It improves the work of additive calculations indirectly. Problems that are difficult to solve in classical case can be solved with incredible ease in here. Every property in Newtonian case can be defined in multiplicative analysis within certain rules.

Many events in nature change exponentially. For example: populations of countries, magnitude of an earthquake [3] are events that behave in this manner. For this reason, using multiplicative analysis instead of usual analysis allows a better physical evaluation of these type events. This calculus also gives better results than usual case in many fields such as finance, economics, biology and demography. A very limited number of studies have been conducted on this analysis until the beginning of the 2000s. Recently, various studies have been carried out on it and quality and effective results have been obtained (see [4]- [13]). A Sturm-Liouville equation is a second-order linear differential equation that allows us to find solutions that form an orthogonal system. While many mathematicians have studied various properties of this equation, some special cases of it produce some special equations [14]- [26]. One of these special equations is the Hermite equations. Hermite equations have many real world practical applications [27]- [33]. We will only focus on the methods of solution and use in a mathematical sense. In solving these equations explicit solutions cannot be found. That is solutions in terms of elementary functions cannot be found. In many cases, it is easier to find a numerical or series solution. Determining the generating function and integral representation of the multiplicative Hermite polynomials, which is one of the main aims of the present study, is very important in physical problems. For example, in the classical case, one computes the bond energy of the atomic nucleus with the use of Eden-Goldstone integral equation which contains Hermite polynomials on the integrals. In addition, the density of the probability distribution of the coordinate in the ensemble of quantum harmonic oscillators is represented by generating function of Hermite polynomials [34]. Therefore, Hermite equation, which has a very important place in classical sense, and its solutions will be dealt with in a multiplicative sense and will be examined in detail. 
In this study, apart from known analysis, Hermite equation will be established and its properties will be examined with methods similar to classical case. The equation we solve in multiplicative case is actually a much more complex equation in classical case, and solutions of the two equations coincide. Before moving on to basic results, let's firstly express the concepts and important theorems of multiplicative analysis that we will use in our study.

Definition 1.1. [4] Let $f: A \subset \mathbb{R} \rightarrow \mathbb{R}$ be differentiable in usual case and $f(x)>0$ for all $x$. If the below limit exists and positive

$$
f^{*}(x)=\lim _{h \rightarrow 0}\left[\frac{f(x+h)}{f(x)}\right]^{\frac{1}{h}}
$$

$f^{*}(x)$ is called multiplicative $\left(\right.$ or $\left.^{*}\right)$ derivative of $f$ at $x$.

Lemma 1.2. [4] Let $f: A \rightarrow \mathbb{R}$ be positive and usual differentiable at $x$. Then, there is following relation between classical and ${ }^{*}$ derivatives.

$$
f^{*}(x)=e^{(\ln o f)^{\prime}(x)}
$$

Theorem 1.3. [4] Let $f, h$ be ${ }^{*}$ differentiable and $p$ be usual differentiable at $x$. The following expressions are provided for * derivative.
i. $(c f)^{*}(x)=f^{*}(x), c \in \mathbb{R}^{+}$,
ii. $(f g)^{*}(x)=f^{*}(x) g^{*}(x)$,
iii. $(f / g)^{*}(x)=f^{*}(x) / g^{*}(x)$,
iv. $\left(f^{h}\right)^{*}(x)=f^{*}(x)^{h(x)} f(x)^{h^{\prime}(x)}$,
v. $(f \circ h)^{*}(x)=f^{*}(h(x))^{h^{\prime}(x)}$,
vi. $(f+g)^{*}(x)=f^{*}(x)^{\frac{f(x)}{f(x)+g(x)}} g^{*}(x)^{\frac{g(x)}{f(x)+g(x)}}$.

Since multiplicative integration will emerge while obtaining Hermite polynomials for multiplicative Hermite equation, let's express fundamental properties of multiplicative integration.

Definition 1.4. [4] Let $f$ be a positive, bounded function on $[a, b]$ where $-\infty<a<b<\infty$. Then, the symbol $\int_{a}^{b} f(x)^{d x}$ is called multiplicative integral or ${ }^{*}$ integral of $f$ on $[a, b]$. By this definition, if $f$ is positive and Riemann integrable on $[a, b]$, then it is *integrable on $[a, b]$ and

$$
\int_{a}^{b} f(x)^{d x}=e^{\int^{b}(\ln o f)(x) d x}
$$

Conversely, one can show that if $f$ is Riemann integrable on $[a, b]$, then

$$
\int_{a}^{b} f(x) d x=\ln \int_{a}^{b}\left(e^{f(x)}\right)^{d x}
$$

Theorem 1.5. [4] Let $f, g>0$ be bounded, ${ }^{*}$ integrable and $h>0$ be usual differentiable on $[a, b]$. Then, below expressions hold:

i. $\int_{a}^{b}\left[f(x)^{k}\right]^{d x}=\left[\int_{a}^{b} f(x)^{d x}\right]^{k}$,

ii. $\int_{a}^{b}[f(x) g(x)]^{d x}=\int_{a}^{b} f(x)^{d x} \int_{a}^{b} g(x)^{d x}$,

iii. $\int_{a}^{b}\left[\frac{f(x)}{g(x)}\right]^{d x}=\frac{\int_{a}^{b} f(x)^{d x}}{\int_{a}^{b} g(x)^{d x}}$,

iv. $\int_{a}^{b} f(x)^{d x}=\int_{a}^{c} f(x)^{d x} \int_{c}^{b} f(x)^{d x}$

v. $\int_{a}^{b}\left[f^{*}(x)^{g(x)}\right]^{d x}=\frac{f(b)^{g(b)}}{f(a)^{g(a)}}\left\{\int_{a}^{b}\left[f(x)^{g^{\prime}(x)}\right]^{d x}\right\}^{-1}$,

where $k \in \mathbb{R}$ is a constant and $c \in[a, b]$. The expression $v$ is known as ${ }^{*}$ integration by parts formula.

In order to avoid any difficulties in expressing main parts of study, inner product function will be defined and inner product space used throughout study will be given in multiplicative case. 
Definition 1.6. Let $X$ be a non empty set and $<,>_{*}: X \times X \rightarrow \mathbb{R}^{+}$be a function such that below axioms hold for $\forall x, y, z \in X$ i. $\langle x, x\rangle_{*} \geq 1$,

ii. $\langle x, x\rangle_{*}=1$ if and only if $x=1$,

iii. $\left.\left.\langle x \oplus y, z\rangle_{*}=<x, z\right\rangle_{*} \oplus<y, z\right\rangle_{*}$,

iv. $<e^{\alpha} \odot x, y>_{*}=e^{\alpha} \odot<x, y>_{*}, \alpha \in \mathbb{R}$,

v. $\langle x, y\rangle_{*}=<y, x>_{*}$.

Then, $\left(X,<,>_{*}\right)$ is called ${ }^{*}$ inner product space and $<,>_{*}$ is ${ }^{*}$ inner product on $X$.

Lemma 1.7. $L_{2}^{*}[a, b]=\left\{f: \int_{a}^{b}[f(x) \odot f(x)]^{d x}<\infty\right\}$ is an *inner product space with

$$
<,>_{*}: L_{2}^{*}[a, b] \times L_{2}^{*}[a, b] \rightarrow \mathbb{R}^{+}, \quad<f, h>_{*}=\int_{a}^{b}[f(x) \odot h(x)]^{d x}
$$

where $f, h \in L_{2}^{*}[a, b]$ are positive functions.

Proof. Proof can be easily demonstrated using properties of multiplicative inner product and definition of given space. $n-$ th order linear homogeneous multiplicative differential equation is denoted by

$$
\left(y^{*(n)}\right)\left(y^{*(n-1)}\right)^{a_{n-1}(x)} \cdots\left(y^{* *}\right)^{a_{2}(x)}\left(y^{*}\right)^{a_{1}(x)} y^{a_{0}(x)}=1
$$

where $y^{*(n)}(x)=e^{(\ln \circ f)^{(n)}(x)}$ and $a_{n-1}(x), \cdots, a_{2}(x), a_{1}(x), a_{0}(x)$ are functions of $x$ [35].

Definition 1.8. [35] Let $x_{0} \in[a, b], N\left(x_{0}\right)$ be a neighbourhood of $x_{0}$ and $f(x)$ be a real function defined on $[a, b]$. In this case $f(x)$ is said to multiplicative-analytic at $x_{0}$ if $f(x)$ can be expressed as a series of natural powers of $\left(x-x_{0}\right)$ for all $x \in N\left(x_{0}\right)$. In other words, $f(x)$ can be expressed as following:

$$
f(x)=\prod_{n=0}^{\infty}\left(c_{n}\right)^{\left(x-x_{0}\right)^{n}},\left(c_{n} \in \mathbb{R}^{+}\right) .
$$

There exists $\delta>0$ such that this series is convergent for all $x$ satisfying $\left|x-x_{0}\right|<\delta$ and divergent for $\left|x-x_{0}\right|>\delta$. $\delta$ is the radius of convergence of the series.

Definition 1.9. [35] Let $x_{0} \in[a, b]$ and functions $a_{k}(x)$ be multiplicative-analytic at $x_{0} \in[a, b]$ for $k=0,1,2, \ldots,(n-1)$. In this case, the point $x_{0} \in[a, b]$ is said to be a multiplicative-ordinary point of (1.1). If a point $x_{0} \in[a, b]$ is not a multiplicativeordinary point, then it is said to be multiplicative singular.

Theorem 1.10. [35] Let $p(x), q(x)$ be analytic functions such as

$$
\begin{array}{ll}
p(x)=\sum_{k=0}^{\infty} p_{k}\left(x-x_{0}\right)^{k}, \quad & \left(t \in\left[x_{0}, x_{0}+\delta_{1}\right] ; \delta_{1}>0\right), \\
q(x)=\sum_{k=0}^{\infty} q_{k}\left(x-x_{0}\right)^{k}, \quad & \left(t \in\left[x_{0}, x_{0}+\delta_{2}\right] ; \delta_{2}>0\right)
\end{array}
$$

and let $x_{0}$ be a multiplicative-ordinary point of the equation

$$
y^{* *}\left(y^{*}\right)^{p(x)} y^{q(x)}=1
$$

Then, there exists a solution to (1.2) as

$$
y=\prod_{k=0}^{\infty} c_{k}^{\left(x-x_{0}\right)^{k}}
$$

for $t \in\left(x_{0}, x_{0}+\rho\right)$ with $\rho=\min \left\{\delta_{1}, \delta_{2}\right\}$ and initial conditions $y\left(x_{0}\right)=c_{0}, y^{*}\left(x_{0}\right)=c_{1}$.

The rest of this study is organized as follows: In the second section, we reconstruct multiplicative Hermite equation by multiplicative Sturm-Liouville equation. A new asymptotic formula for eigenfunction of multiplicative Hermite equation is established by series technique. Moreover, for multiplicative Hermite equation, a generator function is obtained and an integral representation is constructed. In the last section, some spectral properties of multiplicative Hermite equation are examined. 


\section{Multiplicative Hermite equation}

Here, multiplicative Hermite equation will be established from multiplicative Sturm-Liouville equation by some algebraic structures and Hermite polynomials of constructed problem will be obtained. That way, let's express multiplicative algebraic structures that we will encounter while establishing and solving multiplicative Hermite equation. Arithmetic operations created with exponential functions are called multiplicative algebraic operations. Let's show some properties of these operations with a multiplicative arithmetic table for $x, y \in \mathbb{R}^{+}$:

$$
x \ominus y=\frac{x}{y}, \quad x \oplus y=x y, \quad x \odot y=x^{\ln y}=y^{\ln x} .
$$

These operations create some algebraic structures. If $\oplus: A \times A \rightarrow A$ is an operation where $A \neq \phi$ and $A \subset \mathbb{R}^{+}$, algebraic structure $(A, \oplus)$ is called multiplicative group. Similarly, $(A, \oplus, \odot)$ is a multiplicative ring. This situation gives us the opportunity to use these processes easily and define different structures [36].

Let

$$
\left\{\frac{d^{*}}{d x}\left(e^{p(x)} \odot \frac{d^{*} y}{d x}\right)\right\} \oplus\left(e^{q(x)} \odot y\right) \oplus\left(e^{\lambda w(x)} \odot y\right)=1,
$$

be multiplicative Sturm-Liouville equation where $p, q, w$ are real valued, continuous functions [37]. Here, if $p, q, w$ are chosen in a special way as $p(x)=w(x)=e^{-x^{2}}, q(x)=0$, the equation (2.1) transforms into

$$
L[y]=\left[\left(y^{*}(x)\right)^{e^{-x^{2}}}\right]^{*} y^{\lambda e^{-x^{2}}}=1, x \in \mathbb{R}
$$

or

$$
L[y]=y^{* *}\left(y^{*}\right)^{-2 x} y^{\lambda}=1, x \in \mathbb{R},
$$

where $a, b \in \mathbb{R}$ and, $\lambda$ is a spectral parameter. If it is set $\lambda=2 n,(2.2)$ is called multiplicative Hermite equation [35]. Here, $y(x, \lambda)$ is solution of above equation which is called multiplicative Hermite polynomial. Although (2.2) has no multiplicative singular points, it is multiplicative singular Sturm-Liouville equation because of the range for which $x$ is defined. $x=0$ is a multiplicative ordinary point. In [35], the solutions on the neighborhood of this point are found as

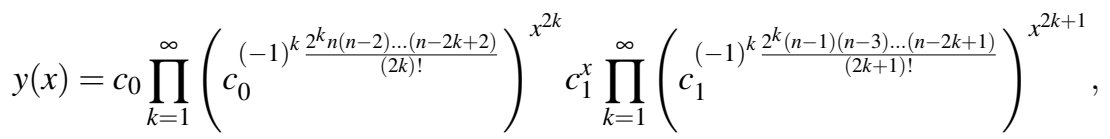

where $c_{0}$ and $c_{1}$ are arbitrary constants. The equation (2.2) will be considered together with the following condition:

$$
\lim _{x \rightarrow \pm \infty} e^{e^{-x^{2}}} y(x, \lambda)=1
$$

The equation (2.2) corresponds to following nonlinear differential equation in classical case. The spectral properties of multiplicative Hermite equation coincide with properties of this nonlinear equation.

$$
\left[y^{\prime \prime}-2 x y^{\prime}+\lambda y\right] y-\left[\left(y^{\prime}\right)^{2}+\lambda y^{2}(1-\ln y)\right]=0 .
$$

According to the cases where $n$ is even and odd, let's define $\widetilde{H}_{n}(x)$ as follows

$$
\widetilde{H}_{n}(x)=\prod_{k=0}^{\left[\frac{n}{2}\right]} e^{\frac{(-1)^{k}(n) !}{k !(n-2 k) !}(2 x)^{n-2 k}}
$$

where

$$
\left[\begin{array}{l}
n \\
2
\end{array}\right]=\left\{\begin{array}{c}
\frac{n}{2}, \text { if } \quad n \text { is even } \\
\frac{n-1}{2}, \text { if } \quad n \text { is odd }
\end{array} .\right.
$$

Eigenfunctions $\widetilde{H}_{n}(x)$ are $n$-th degree multiplicative Hermite polynomials. These numbers and polynomials play an important role in various areas of mathematics and physics, including numerical theory, combinations, special functions, and differential equations. Many interesting properties about them have been explored. For example, in mathematics and physics, the Hermite polynomials are a classical orthogonal polynomial sequence. In probability, they appears as the Edgeworth series; in combinatorics, they arise in the umbral calculus as an example of an Appell sequence; in numerical analysis, they play a role in Gaussian quadrature; and in physics, they give rise to the eigenstates of the quantum harmonic oscillator. The polynomials arise in signal processing as Hermitian wavelets for wavelet transform analysis.

Now, let's obtain a generator function for multiplicative Hermite equation and find integral representations of multiplicative Hermite polynomial: 
Lemma 2.1. The generator functions of multiplicative Hermite polynomials have the following representation:

$$
H(x, t)=e^{e^{2 x t-t^{2}}}=\prod_{n=0}^{\infty}\left\{\widetilde{H}_{n}(x)\right\}^{\frac{t^{n}}{n !}}
$$

where $|x|<\infty,|t|<\infty$ and $\widetilde{H}_{n}(x)$ is defined as (2.4).

Proof. Consider the following function

$$
H(x, t)=e^{e^{2 x t-t^{2}}}=e^{\left[\sum_{m=0}^{\infty} \frac{(2 x t)^{m}}{m !}\right]\left[\sum_{k=0}^{\infty} \frac{\left(-t^{2}\right)^{k}}{k !}\right]}=\prod_{n=0}^{\infty}\left\{\widetilde{H}_{n}(x)\right\}^{\frac{t^{n}}{n !}} .
$$

If it is proven that $\widetilde{H}_{n}(x)$ is the multiplicative Hermite polynomial (that is, it satisfies the equation (2.2)), the proof completes. Firstly, ${ }^{*}$ differentiating (2.5) with respect to $x$, we get

$$
e^{2 t e^{2 x t-t^{2}}}=\prod_{n=1}^{\infty}\left\{\widetilde{H}_{n}^{*}(x)\right\}^{\frac{t^{n}}{n !}}
$$

or

$$
\prod_{n=0}^{\infty}\left\{\widetilde{H}_{n}(x)\right\}^{\frac{2 t^{n+1}}{n !}}=\prod_{n=1}^{\infty}\left\{\widetilde{H}_{n}^{*}(x)\right\}^{\frac{t^{n}}{n !}} .
$$

From the last equality, if the exponents of $t$ are compared, we obtain the recurrence relation

$$
\widetilde{H}_{n+1}^{*}(x)=\left\{\widetilde{H}_{n}(x)\right\}^{2(n+1)} .
$$

Secondly, ${ }^{*}$ differentiating (2.5) with respect to $t$, and then comparing the exponents of $t$, we have the other recurrence relation

$$
\widetilde{H}_{n+1}(x)\left\{\widetilde{H}_{n}(x)\right\}^{-2 x}\left\{\widetilde{H}_{n-1}(x)\right\}^{2 n}=1 .
$$

Thirdly, ${ }^{*}$ differentiating (2.7) with respect to $x$, we have

$$
\widetilde{H}_{n+1}^{*}(x)\left\{\widetilde{H}_{n}^{*}(x)\right\}^{-2 x}\left\{\widetilde{H}_{n}(x)\right\}^{-2}\left\{\widetilde{H}_{n-1}^{*}(x)\right\}^{2 n}=1 .
$$

Using (2.6) in the last equation, we take

$$
\left\{\widetilde{H}_{n}(x)\right\}^{2(n+1)}\left\{\widetilde{H}_{n}^{*}(x)\right\}^{-2 x}\left\{\widetilde{H}_{n}(x)\right\}^{-2} \widetilde{H}_{n}^{* *}(x)=1
$$

or

$$
\widetilde{H}_{n}^{* *}(x)\left\{\widetilde{H}_{n}^{*}(x)\right\}^{-2 x}\left\{\widetilde{H}_{n}(x)\right\}^{2 n}=1 .
$$

This last equality shows that $\widetilde{H}_{n}(x)$ satisfies the equation (2.2).

The following lemma has been proved by [35] using a different technique. Here, the proof is made using generator functions.

Lemma 2.2. The multiplicative Rodrigues formula for $\widetilde{H}_{n}(x)$ has the formula

$$
\widetilde{H}_{n}(x)=\left[\frac{d^{*(n)}}{d x^{n}} e^{e^{-x^{2}}}\right]^{(-1)^{n} e^{x^{2}}}, \quad n=0,1,2, \cdots
$$


Proof. From the formula (2.5), we obtain

$$
e^{e^{\left(x^{2}-(t-x)^{2}\right)}}=\prod_{n=0}^{\infty}\left\{\widetilde{H}_{n}(x)\right\}^{t^{n}}
$$

or

$$
\left\{e^{e^{-(t-x)^{2}}}\right\}^{x^{x^{2}}}=\left\{\widetilde{H}_{0}(x)\right\}^{\frac{1}{0 !}}\left\{\widetilde{H}_{1}(x)\right\}^{\frac{t}{! !}}\left\{\widetilde{H}_{2}(x)\right\}^{\frac{t^{2}}{2 !}} \ldots\left\{\widetilde{H}_{n}(x)\right\}^{\frac{t^{n}}{n !}}\left\{\widetilde{H}_{n+1}(x)\right\}^{\frac{t^{n+1}}{(n+1) !}} \ldots
$$

By taking $n$-times *derivative of both side of (2.9) with respect to $t$, we get

$$
\left\{\frac{\partial^{*(n)}}{\partial t^{n}} e^{e^{-(t-x)^{2}}}\right\}^{e^{x^{2}}}=1.1 \ldots\left\{\widetilde{H}_{n}(x)\right\}^{\frac{n !}{n !}}\left\{\widetilde{H}_{n+1}(x)\right\}^{\frac{(n+1) !}{(n+1) !} t} \ldots
$$

Then,

$$
\widetilde{H}_{n}(x)=\left.\left\{\frac{\partial^{*(n)}}{\partial t^{n}} e^{e^{-(t-x)^{2}}}\right\}^{e^{x^{2}}}\right|_{t=0}
$$

for $t=0$. After some basic calculations, taking the relation

$$
\left.\left\{\frac{\partial^{*(n)}}{\partial t^{n}} e^{e^{-(t-x)^{2}}}\right\}\right|_{t=0}=\left\{\frac{d^{*(n)}}{d x^{n}} e^{e^{-x^{2}}}\right\}^{(-1)^{n}}
$$

we prove (2.8).

Lemma 2.3. The integral representation of multiplicative Hermite polynomials is as follows:

$$
\widetilde{H}_{n}(x)=\left\{\int_{-\infty}^{\infty}\left\{e^{(x+i s)^{n} e^{-s^{2}}}\right\}^{d s}\right\}^{\frac{2^{n}}{\sqrt{\pi}}} .
$$

Proof. Consider the integral representation of $e^{e^{-x^{2}}}$ as:

$$
e^{e^{-x^{2}}}=\left\{\int_{-\infty}^{\infty}\left(e^{e^{-s(s+2 i x)^{2}}}\right)^{d s}\right\}^{\frac{1}{\sqrt{\pi}}} .
$$

Taking the $n$-th *derivative of (2.10) with respect to $x$, we have

$$
\begin{aligned}
\frac{d^{*}(n)}{d x^{n}}\left(e^{e^{-x^{2}}}\right) & =\left\{\int_{-\infty}^{\infty}\left(e^{(-i 2 s)^{n} e^{-s(s+2 i x)}}\right)^{d s}\right\}^{\frac{1}{\sqrt{\pi}}} \\
& =\left\{\int_{-\infty}^{\infty}\left(e^{(i s)^{n} e^{-(s+i x)^{2}}}\right)^{d s}\right\}^{(-1)^{n} \frac{2^{n}}{\sqrt{\pi}} e^{-x^{2}}} \\
& =\left\{\int_{-\infty}^{\infty}\left(e^{(x+i s)^{n} e^{-s^{2}}}\right)^{d s}\right\}^{(-1)^{n} \frac{2^{n}}{\sqrt{\pi}} e^{-x^{2}}} .
\end{aligned}
$$

By considering the $(-1)^{n} e^{x^{2}}$ power of both sides for (2.11), we get

$$
\left\{\frac{d^{*(n)}}{d x^{n}}\left(e^{e^{-x^{2}}}\right)\right\}^{(-1)^{n} e^{x^{2}}}=\left\{\int_{-\infty}^{\infty}\left(e^{(x+i s)^{n} e^{-s^{2}}}\right)^{d s}\right\}^{\frac{2^{n}}{\sqrt{\pi}}} .
$$

From (2.8), proof is completed. 
Now, let's express some special cases of multiplicative Hermite polynomials, which have an important place in applications.

Remark 2.4. [35] By (2.4), some multiplicative Hermite polynomials are as follows:

$$
\begin{aligned}
\widetilde{H}_{0}(x) & =e, \\
\widetilde{H}_{1}(x) & =e^{2 x}, \\
\widetilde{H}_{2}(x) & =e^{4 x^{2}-2}, \\
\widetilde{H}_{3}(x) & =e^{8 x^{3}-12 x}, \\
\widetilde{H}_{4}(x) & =e^{16 x^{4}-48 x^{2}+12}, \\
\widetilde{H}_{5}(x) & =e^{32 x^{5}-160 x^{3}+120 x}
\end{aligned}
$$

Remark 2.5. Multiplicative Hermite polynomials provide the following properties:

i. $\widetilde{H}_{n}(x)=e^{H_{n}(x)}$,

ii. $\widetilde{H}_{n}(-x)=\left[\widetilde{H}_{n}(x)\right]^{(-1)^{n}}, \quad \widetilde{H}_{n}^{*}(-x)=\left[\widetilde{H}_{n}^{*}(x)\right]^{(-1)^{n+1}}$,

iii. $\widetilde{H}_{2 n}(0)=e^{(-1)^{n} \frac{(2 n) !}{n !}}, \quad \widetilde{H}_{2 n+1}(0)=1$,

iv. $\widetilde{H}_{2 n}^{*}(0)=1, \quad \widetilde{H}_{2 n+1}^{*}(0)=e^{(-1)^{n} \frac{(2 n+2) !}{(n+1) !}}$,

for $n=0,1,2, \ldots$

Proof. From multiplicative Rodrigues formula, the proofs of these features can be easily made similar to the classical situation.

\section{Some spectral properties of multiplicative Hermite problem}

In this section, we consider the problem (2.2)-(2.3). As will be remembered from the previous section, general solution of the equation (2.2) is represented (2.4).

Now, let's express some spectral properties of multiplicative Hermite polynomial. The following lemma has been proved by [35] with different perspective. Here, the proof is made using multiplicative Sturm-Liouville equation.

Lemma 3.1. The multiplicative Hermite polynomials $\widetilde{H}_{n}(x)$ and $\widetilde{H}_{m}(x)$ are orthogonal according to the weight function $e^{e^{-x^{2}}}$ on $(-\infty, \infty)$ for $m \neq n$. Furthermore,

$$
\int_{-\infty}^{\infty}\left[e^{e^{-x^{2}}} \odot \widetilde{H}_{n}(x) \odot \widetilde{H}_{m}(x)\right]^{d x}=\left\{\begin{array}{cc}
1, & \text { if } m \neq n \\
e^{2^{n} n ! \sqrt{\pi}}, & \text { if } m=n
\end{array} .\right.
$$

Proof. Let's do the proof separately for two cases.

i. Let $m \neq n$. Since multiplicative Hermite polynomials $\widetilde{H}_{n}(x)$ and $\widetilde{H}_{m}(x)$ are solutions of the equation (2.2), we can write

$$
\begin{gathered}
{\left[\left(\widetilde{H}_{n}^{*}(x)\right)^{e^{-x^{2}}}\right]^{*}\left\{\widetilde{H}_{n}(x)\right\}^{2 n e^{-x^{2}}}=1,} \\
{\left[\left(\widetilde{H}_{m}^{*}(x)\right)^{e^{-x^{2}}}\right]^{*}\left\{\widetilde{H}_{m}(x)\right\}^{2 m e^{-x^{2}}}=1 .}
\end{gathered}
$$

Let us take $\ln \widetilde{H}_{m}(x)$ and $\ln \widetilde{H}_{n}(x)$-th powers of (3.1) and (3.2) respectively. Then, if we use multiplicative integration to both sides on $(-\infty, \infty)$ after the obtained relations are divided by side, we get

$$
\left[\int_{-\infty}^{\infty}\left\{\left(\widetilde{H}_{n}^{\ln \widetilde{H}_{m}}\right)^{e^{-x^{2}}}\right\}^{d x}\right]^{2(m-n)}=\int_{-\infty}^{\infty}\left[\frac{d^{*}}{d x}\left(W\left(\widetilde{H}_{m}, \widetilde{H}_{n}\right)\right)^{e^{-x^{2}}}\right]^{d x},
$$

where $W\left(\widetilde{H}_{m}, \widetilde{H}_{n}\right)=\left(\widetilde{H}_{m} \odot \widetilde{H}_{n}^{*}\right) \ominus\left(\widetilde{H}_{m}^{*} \odot \widetilde{H}_{n}\right)$. Since $m \neq n$ and the conditions (2.3), it gives

$$
\int_{-\infty}^{\infty}\left\{\left(\widetilde{H}_{n}(x) \odot \widetilde{H}_{m}(x)\right)^{e^{-x^{2}}}\right\}^{d x}=1 .
$$


So, the proof for $m \neq n$ is completed.

ii. Let $m=n$. After taking $n \rightarrow n-1$ in the equation (2.7), circle multiplying the equation (2.7) by $e^{e^{-x^{2}}} \odot \widetilde{H}_{n}(x)$, we have

$$
\left\{e^{e^{-x^{2}}} \odot \widetilde{H}_{n}(x) \odot \widetilde{H}_{n}(x)\right\}\left\{\widetilde{H}_{n}(x) \odot \widetilde{H}_{n-1}(x)\right\}^{-2 x e^{-x^{2}}}\left\{\widetilde{H}_{n}(x) \odot \widetilde{H}_{n-2}(x)\right\}^{2(n-1) e^{-x^{2}}}=1 .
$$

Then, if we use (3.3) after *integrating to both sides of the last equation on $(-\infty, \infty)$, we get

$$
\int_{-\infty}^{\infty}\left\{\left(\widetilde{H}_{n}(x) \odot \widetilde{H}_{n}(x)\right)^{e^{-x^{2}}}\right\}^{d x} \int_{-\infty}^{\infty}\left\{\left(\widetilde{H}_{n}(x) \odot \widetilde{H}_{n-1}(x)\right)^{-2 x e^{-x^{2}}}\right\}^{d x}=1 .
$$

On the other hand, let's circle multiply the equation (2.7) by $e^{e^{-x^{2}}} \odot \widetilde{H}_{n-1}(x)$, then, if we use (3.3) after *integrating both sides of this equation on $(-\infty, \infty)$, we have

$$
\int_{-\infty}^{\infty}\left\{\left(\widetilde{H}_{n-1}(x) \odot \widetilde{H}_{n-1}(x)\right)^{2 n e^{-x^{2}}}\right\}^{d x} \int_{-\infty}^{\infty}\left\{\left(\widetilde{H}_{n}(x) \odot \widetilde{H}_{n-1}(x)\right)^{-2 x e^{-x^{2}}}\right\}^{d x}=1 .
$$

Considering (3.4) and (3.5) together, the following recurrence relation is obtained:

$$
\int_{-\infty}^{\infty}\left\{\left(\widetilde{H}_{n}(x) \odot \widetilde{H}_{n}(x)\right)^{e^{-x^{2}}}\right\}^{d x}=\int_{-\infty}^{\infty}\left\{\left(\widetilde{H}_{n-1}(x) \odot \widetilde{H}_{n-1}(x)\right)^{2 n e^{-x^{2}}}\right\}^{d x}
$$

After taking $n=1,2,3, \ldots$ and using $\int_{-\infty}^{\infty}\left\{e^{e^{-x^{2}}}\right\}^{d x}=e^{\sqrt{\pi}}$, gives

$$
\int_{-\infty}^{\infty}\left\{\left(\widetilde{H}_{n}(x) \odot \widetilde{H}_{n}(x)\right)^{e^{-x^{2}}}\right\}^{d x}=e^{2^{n} n ! \sqrt{\pi}} .
$$

It completes the proof.

Lemma 3.2. *eigenvalues of the problem (2.2)-(2.3) are all real.

Proof. Let $\lambda$ be a complex eigenvalue for $y(x, \lambda)$. Then, $\bar{\lambda}$ is eigenvalue corresponding to $\overline{y(x, \lambda)}$. By (3.3),

$$
\left\{\int_{-\infty}^{\infty}\left\{\left(y^{\ln \bar{y}}\right)^{e^{-x^{2}}}\right\}^{d x}\right\}^{\lambda-\bar{\lambda}}=1 .
$$

By the notion of multiplicative integration,

$$
\left\{\int_{-\infty}^{\infty} e^{-x^{2}}|\ln y|^{2} d x\right\}^{\lambda-\bar{\lambda}}=0
$$

Since $y$ must be a non-trivial solution, i.e. $y \neq 1$, and $\int_{-\infty}^{\infty} e^{-x^{2}}|\ln y|^{2} d x>0$, we get $\lambda=\bar{\lambda}$. This completes the proof.

Self-adjoint operators are used in functional analysis and quantum mechanics. In quantum mechanics their importance lies in which physical observables such as position, momentum, angular momentum and spin are represented by self-adjoint operators. Because of this importance, we examine the self-adjointness of the multiplicative Hermite operator.

Lemma 3.3. Multiplicative Hermite operator L on (2.2) is self-adjoint in $L_{2}^{*}(\mathbb{R})$.

Proof. Assume that $u$ and $v$ are positive multiplicative Hermite polynomials on $\mathbb{R}$ and $u, v \in C^{*(2)}$ where $C^{*(2)}$ is the set of all functions whose second order multiplicative derivatives are continuous. By the definition of multiplicative Hermite operator and multiplicative derivative, we get

$$
\frac{(L v)^{\ln u}}{(L u)^{\ln v}}=\frac{d^{*}}{d x}\left[(W(u, v))^{e^{-x^{2}}}\right]
$$


By *integrating both sides of (3.6) on $\mathbb{R}$,

$$
\int_{-\infty}^{\infty}\left[\frac{(L v)^{\ln u}}{(L u)^{\ln v}}\right]^{d x}=\frac{\lim _{x \rightarrow-\infty}(W(u, v))^{e^{-x^{2}}}}{\lim _{x \rightarrow \infty}(W(u, v))^{e^{-x^{2}}}}
$$

and using the properties of limit, we acquire

$$
<L u, v>_{*}=<u, L v>_{*}
$$

This indicates that the given multiplicative Hermite operator is self-adjoint on $L_{2}^{*}(\mathbb{R})$.

\section{Conclusion}

Hermite's differential equation is frequently encountered in physics and engineering. It arises in numerous problems, particularly in boundary value problems for spheres. Because Hermite polynomials with these equations and their solutions have such an important place, we carried these concepts to multiplicative analysis. First, we set up Hermite equation in multiplicative analysis. Then, we obtained multiplicative Hermite polynomials for different situations using multiplicative series methods. Finally, we examined some spectral properties of these multiplicative polynomials. In fact, these investigations coincide with spectral properties of a much more complex nonlinear equation in classical case.

\section{Acknowledgements}

The authors would like to express their sincere thanks to the editor and the anonymous reviewers for their helpful comments and suggestions.

\section{Funding}

There is no funding for this work.

\section{Availability of data and materials}

Not applicable.

\section{Competing interests}

The authors declare that they have no competing interests.

\section{Author's contributions}

All authors contributed equally to the writing of this paper. All authors read and approved the final manuscript.

\section{References}

[1] M. Grossman, An introduction to Non-Newtonian calculus, Int. J. Math. Educ. Sci. Technol., 10(4) (1979) $525-528$.

[2] M. Grossman, R. Katz, Non-Newtonian Calculus, Lee Press, Pigeon Cove, MA, 1972.

[3] A. Benford, The Law of anomalous numbers, Proc. Am. Philos. Soc., 78 (1938) 551-572.

[4] A. E. Bashirov, E. M. Kurpınar, A. Özyapıcı, Multiplicative calculus and its applications, J. Math. Anal. Appl., 337 (2008), 36-48.

[5] A. E. Bashirov, M. Riza, On complex multiplicative differentiation, TWMS J. Apl. \& Eng. Math., 1(1) (2011) 75-85.

[6] A. E. Bashirov, E. Misırlı, Y. Tandogdu, A. Özyapıcı, On modeling with multiplicative differential equations, Appl. Math. J. Chinese Univ. Ser. A, 26(4) (2011) 425-438

[7] K. Boruah, B. Hazarika, G-Calculus, TWMS J. Apl. \& Eng. Math., 8(1) (2018), 94-105.

[8] L. Florack, Hv. Assen, Multiplicative calculus in biomedical image analysis, J. Math. Imaging Vis., 42 (2012), 64-75.

[9] R. A. Guenther, Product integrals and sum integrals, Int. J. Math. Educ. Sci. Technol., 14(2) (1983), $243-249$.

[10] A. Slavík, Product Integration, Its History and Applications, Matfyzpress, Prague, 2007.

[11] D. Stanley, A multiplicative calculus, Primus, IX (4) (1999), 310-326.

[12] S. Goktas, E. Yilmaz, A. C. Yar, Multiplicative derivative and its basic properties on time scales, Math. Methods Appl. Sci., (2021), 310-326, https://doi.org/10.1002/mma.7910

[13] E. Yilmaz, Multiplicative Bessel equation and its spectral properties, Ric. Mat., (2021), 1-17. https://doi.org/10.1007/s11587-021-00674-1

[14] L. C. Andrews, Special Functions of Mathematics for Engineers, SPIE, USA, 1998.

[15] X. Duan, Spectral Theory of the Hermite Operator on $L^{p}\left(R^{n}\right)$, Math. Model. Nat. Phenom., 9 (2014) 39-43.

[16] W. N. Everitt, L. L. Littlejohn, R. Wellman, The left-definite spectral theory for the classical Hermite differential equation, J. Comput. Appl. Math., 121 (2000), 313-330.

[17] G. Freiling, V. Yurko, Inverse problems for differential operators with singular boundary conditions, Math. Nachr., 278(12-13) (2005) 1561-1578.

[18] T. Gulsen, E. Yilmaz, E. S. Panakhov, On a lipschitz stability problem for p-Laplacian Bessel equation, Commun. Fac. Sci. Univ. Ank. Ser. A1. Math. Stat., 66 (2017) 253-262.

[19] T. Gulsen, E. Yilmaz, M. Hamadamen, Inverse nodal problem for p-Laplacian Bessel equation with polynomially dependent spectral parameter, Demonstr. Math., 51 (2018) 255-263.

[20] E. Hasanov, G. Uzgoren, A. Buyukaksoy, Diferensiyel Denklemler Teorisi, Papatya Yayıncılık, İstanbul, 2002.

[21] Y. He, F. Yang, Some recurrence formulas for the Hermite polynomials and their squares, Open Math., 16 (2018) 553-560. 
[22] K. W. Hwang, C. S. Ryoo, Differential equations associated with two variable degenerate Hermite polynomials, Mathematics, 8 (2020) 228-245.

[23] A. M. Krall, Spectral analysis for the generalized Hermite polynomials, Trans. Amer. Math. Soc., 344 (1994) $155-172$.

[24] V. A. Marchenko, Sturm-Liouville Operators and Their Applications, Kiev: Naukova Dumka, 1977. Russian; English, translation, Birkhauser, 1986.

[25] P. N. Sadjang, W. Koepf, M. Foupouagnigni, On moments of classical orthogonal polynomials, J. Math. Anal. Appl., 424 (2015) $122-151$.

[26] R. Yilmazer, Discrete fractional solutions of a Hermite equation, J. Inequal. Appl., 10(1) (2019), 53-59.

[27] A. Shehata, R. Bhukya, Some properties of Hermite matrix polynomials, J. Int. Math. Virtual Inst., 5 (2015), 1-17.

[28] L. M. Upadhyaya, A. Shehata, A new extension of generalized Hermite matrix polynomials, Bull. Malays. Math. Sci. Soc., 38(1) (2015), 165-179.

[29] A. Shehata, Connections between Legendre with Hermite and Laguerre matrix polynomials, Gazi Univ. J. Sci., 28(2) (2015), 221-230.

[30] A. Shehata, B. Cekim, Some relations on Hermite-Hermite matrix polynomials, Politehn. Univ. Bucharest Sci. Bull. Ser. A Appl. Math. Phys., 78(1) (2016), 181-194.

[31] A. Shehata, L. M. Upadhyaya, Some relations satisfied by Hermite-Hermite matrix polynomials, Math. Bohem., 142(2) (2017), 145-162.

[32] A. Shehata, On new extensions of the generalized Hermite matrix polynomials, Acta Comment. Univ. Tartu. Math., 22(2) (2018), 203-222.

[33] A. Shehata, Certain properties of generalized Hermite-Type matrix polynomials using Weisner's group theoretic techniques, Bull. Braz. Math. Soc. (N.S.), 50(2) (2019), 419-434.

[34] S. Dvořák, Generating function and integral representation of Hermite polynomials in physical problems, Czechoslovak J. Phys. B, 23 (1973)

[35] N. Yalçın, The solutions of multiplicative Hermite differential equation and multiplicative Hermite polynomials, Rend. Circ. Mat. Palermo (2), 70 (2021) 9-21.

[36] U. Kadak, Y. Gurefe, A generalization on weighted means and convex functions with respect to the Non-Newtonian calculus, Int. J. Anal., Article ID $5416751,(2016)$

[37] S. Goktas, A New Type of Sturm-Liouville equation in the non-Newtonian calculus, J. Funct. Spaces, 5203939 (2021) 1-8. 